\title{
The Role of Primary Care in Asthma Control and Severity
}

\author{
Joel Agarwal, BSc ${ }^{1,2}$, Monette Dimitrov ${ }^{1}$, Kerri MacKay ${ }^{3}$, Alan Kaplan, \\ $M^{4}$, Donald Cockcroft, MD, FRCPC ${ }^{5}$, Dilini Vethanayagam, MD, \\ FRCPC $^{1}$
}

${ }^{1}$ Department of Medicine, University of Alberta, ${ }^{2}$ Department of Biological Sciences, University of Alberta, ${ }^{3}$ Canadian Severe Asthma Network, ${ }^{4}$ Family Practice Airways Group of Canada, ${ }^{5}$ Faculty of Medicine, University of Saskatchewan

Corresponding author: dilini@ualberta.ca

\section{ABSTRACT}

Background: Asthma is a common chronic inflammatory disease of the airways affecting 3 million Canadians. Primary Care Providers (PCPs) are integral to care coordination, enhanced through the development of a strong patient-PCP relationship with Continuity of Care (COC). A recent $\mathrm{CIHI}$ study noted that $40 \%$ of Albertans do not have a COC model for primary care.

Objectives: We aim to evaluate how primary care for adults with asthma impacts different measures of control.

Methods: Prospective population-based recruitment of adults through various community venues across Alberta. Those who had self-reported asthma and were willing to participate completed a survey which included demographics, comorbidities, medication use (including biologics, allergy medications, steroids), Asthma Control Questionnaire (ACQ-5), Asthma Control Test (ACT), Quality of Life (QoL) measured through the mini-Asthma Quality of Life Questionnaire (mini-AQLQ) and health care utilization (including Emergency Department (ED) visits, hospitalizations and ICU stays for asthma).

Results: Of the 1685 individuals approached, 61 (3.6\%) reported having asthma, of which 47 lived in Alberta. Most $(41,87 \%)$ had a PCP, with $30(64 \%)$ visiting their PCP at least twice a year. Uncontrolled asthma was noted in $21(45 \%)$ with either the ACQ-5 or ACT. The mini-AQLQ indicated 5 (11\%) with reduced QoL. Mean lifetime hospitalizations, lifetime Emergency Department (ED) visits, and ICU stays related to asthma were $1.52,4.55$ and 0.25 respectively. Further, mean hospitalizations and ED visits in the past 12 months related to asthma were 0.05 and 0.30 respectively.

Conclusions: Asthma control was poor in 21 (45\%) surveyed individuals, suggesting sub-optimal asthma management in Alberta. Knowledge of Primary Care Networks (PCNs) was low, while ED and hospital usage was high. 


\section{Introduction}

Asthma is a chronic inflammatory condition of the airways which continues to impact the Canadian healthcare system (Reddel, Taylor, Bateman, Boulet, Boushey, Busse, and de Jongste, 2009). National and international asthma guidelines agree that the primary goal of managing asthma is disease control, thereby leading to reduction in exacerbations, health care utilization and morbidity and mortality (Reddel et al., 2009; Lougheed, Lemière, Dell, Ducharme, FitzGerald, Leigh, and Boulet, 2010; Bateman, Hurd, Barnes, Bousquet, Drazen, FitzGerald, and Pizzichini, 2008) In Canada, Primary Care Providers (PCPs) are the entry point to the health care system, and play an integral role in chronic disease management such as asthma (Menec, Sirski, Attawar, and Katz, 2006; Haggerty, Reid, Freeman, Starfield, Adair, and McKendry, 2003; Canadian Institute for Health Information [CIHI], 2015). PCPs in Canada include general practitioners, family physicians, nurse practitioners, pediatricians, and physician assistants, all of whom play important roles (Kaplan, 2008). A key aspect to chronic disease management is having a strong and consistent patient-PCP relationship in primary care, forming the basis of the continuity of care (COC) model (Haggerty et al., 2003; CIHI, 2015). Strong COC is associated with reduced health care costs for individuals with chronic conditions, lower utilization of emergency and hospital services, compliance with education use, as well as fosters depth of relationship in knowledge, trust, loyalty and regard (Menec et al., 2006; Hollander and Kadlec, 2015; Christakis, Mell, Koepsell, Zimmerman, and Connell, 2001; Ridd, Shaw, Lewis, and Salisbury, 2009; Love, MAINOUS, Talbert, and Hager, 2000). Furthermore, in Alberta, PCPs are often linked with Primary Care Networks (PCNs), which provide team-based collaborative efforts in addressing important primary health care needs, such as chronic disease management.
A 2015 report from the Canadian Institute for Health Information (ClHI) noted that $40 \%$ of Albertans do not have access to a $\mathrm{COC}$ model for their primary care (CIHI, 2015). This report identifies a significant proportion of Albertans who may have reduced likelihood for optimal asthma management. Currently, no studies have evaluated asthma control as an outcome of differential access to primary care in Alberta. For an asthma patient with a strong and continual relationship with their PCP, one should see better asthma control which can be manifested as a decrease in hospitalizations, Emergency Department (ED) visits, Intensive Care Unit (ICU) stays, use of systemic steroids, and overall better scores on validated asthma control questionnaires. In theory, many of these should stem from a strong COC model; however, this is not always easy to achieve in practice, especially with differential access to health care across the province. Access to basic health services in many rural areas is often not feasible due to long distances, especially in Alberta where almost $20 \%$ of Alberta's population comprises of individuals living in rural areas, many of which have limited access to primary care facilities (Statistics Canada, 2018).

We aim to evaluate how primary care for adults with asthma impacts different measures of control. We hypothesize that suboptimal primary care and continuity of care would be common for adults with asthma in Alberta and could adversely affect outcomes. This includes hospitalizations and ED visits, ICU stays, medication use, and standardized measures of asthma control.

\section{Methods}

This prospective study involved recruitment of participants in community (non-clinical) venues in Alberta. A secure web-based data collection program (REDCap) was used to collect information in 2015/2016 through this student-led project.

Ethics approval was obtained through the University of Alberta's research ethics board. The 
survey was administered through populationbased recruitment of willing adult (18 or older) participants who had a PCP diagnosis of asthma by self-report as their primary respiratory diagnosis. Those whose residences were outside Alberta were excluded from this study.

Recruitment was done by randomly approaching individuals in community venues such as malls, libraries, coffee shops, and community centres. All adult participants who were willing to participate were asked first if they had asthma, and then if they had physiology testing (spirometry/ pulmonary function test [PFT]) done confirming asthma in the past 12 months. They were also asked if they had ever had airway challenge tests (methacholine or exercise challenge) for asthma diagnosis. Those who answered no to the latter two questions were not excluded from the study, and such information was recorded.

We excluded pediatric subjects (less than 18 years) and those unwilling to participate. Survey recruitment was not performed in clinical and other medical venues (i.e. clinics, pulmonary function laboratories etc.) as to avoid a biased population who may likely receive community asthma care. The survey collected information on demographics, comorbid illnesses, medication use (inhaled, systemic steroids, biologics, and non-airways medications), allergies, and health care utilization (including ED visits, hospitalizations, ICU stays, and neonatalintensive care unit(NICU) stays forasthma).

The survey components included the Asthma Control Questionnaire (ACQ-5) (Juniper, O' byrne, Guyatt, Ferrie, and King, 1999) and Asthma Control Test (ACT) (Nathan, Sorkness, Kosinski, Schatz, Li, Marcus, and Pendergraft, 2004), a validated and standardized method of quantifying asthma control. The participants' quality of life (secondary outcome) was assessed through the mini-Asthma Quality of Life Questionnaire (mini-AQLQ). These questionnaires were created by Juniper et al (1999).
Standardized Questionnaires to Measure Asthma Control:

Differences between the ACQ-5 and the ACT have been discussed in the past, as recall bias may be a factor in documented accuracy of the tools due to the patient recall ( 1 week recall for ACQ; 4 week recall for $\mathrm{ACT}$ ). These questionnaires measure both the adequacy of and change in asthma control, occurring spontaneously or as a result of treatment. The distinguishing feature of the ACT is the use of a criterion measure of asthma control; where the concordance between a patient-based measure of asthma control (shown to be invaluable) and an asthma specialist-based measure of asthma control was evaluated (Nathan et al., 2004; Schatz, Sorkness, Li, Marcus, Murray, Nathan, and Jhingran, 2006; Jia, Zhang, Lv, Liang, Jiang, Powell, H., and Wang, 2013).

Asthma Control Questionnaire:

The ACQ has 7 questions and includes the top scoring 5 symptoms (rated most important by 91 asthma clinicians in 18 countries) consisting of: waking at night by symptoms, waking in the mornings with symptoms, limitation of daily activities, shortness of breath, and wheeze (Juniper et al., 1999). The questionnaire also exists as 3 shortened versions of the 7-item ACQ. Of these, 2 are variations including 6 items (symptoms as well Forced Expiratory Volume (FEV1), or symptoms and $\beta$-agonists). For our study we used the third, which is a 5-item ACQ consisting of symptoms alone and no physiologic testing. There is strong evidence that when the FEV1 and $\beta$-agonist questions are omitted from the original $A C Q$, the results are very similar to those generated by the complete ACQ (Juniper, Svensson, Mörk, and Ståhl, 2005). The shortened versions can be used without loss of validity or change in interpretation, and are useful in situations where it is not feasible to collect airway calibre or $\beta$-agonist data (such as in large populations or epidemiological studies) (Juniper et al., 2005).

\section{Asthma Control Test:}

In addition to the ACQ-5, the ACT has also been validated to assess asthma control. The test 
includes 5 questions (to be answered recalling how their asthma has been in the previous 4 weeks) consisting of: nocturnal, rescue medication and role limitations. The questions are based on a 5-point scale (1=all the time and $5=$ not at all). Additionally, the ACT differs from the ACQ in the use of cut-off points and the minimally important difference values (Goeman, Jenkins, Crane, Paul, and Douglass, 2013)

Table 1. Descriptive summary statistics $(n=47)$

\begin{tabular}{|c|c|}
\hline Variable & n (\%) \\
\hline \multicolumn{2}{|c|}{$\begin{array}{l}\text { Pulmonary Physiology Confirming } \\
\text { Asthma }\end{array}$} \\
\hline Yes & $17(36)$ \\
\hline No & $30(64)$ \\
\hline \multicolumn{2}{|c|}{ Duration of Asthma Diagnosis } \\
\hline$<1$ Year & $4(9)$ \\
\hline 1-5 Years & $4(9)$ \\
\hline$>5$ Years & $39(83)$ \\
\hline \multicolumn{2}{|l|}{ Age Category (\%) } \\
\hline $18-39$ & $29(62)$ \\
\hline $40-65$ & $14(30)$ \\
\hline $66-79$ & $1(2)$ \\
\hline$\geq 80$ & $3(6)$ \\
\hline \multicolumn{2}{|l|}{ ACQ-5 Score (\%) } \\
\hline Uncontrolled & $15(32)$ \\
\hline Controlled & $32(68)$ \\
\hline \multicolumn{2}{|l|}{ ACT Score (\%) } \\
\hline Uncontrolled & $17(36)$ \\
\hline Controlled & $30(64)$ \\
\hline \multicolumn{2}{|l|}{ Mini-AQLQ (\%) } \\
\hline Poor-moderate & $5(11)$ \\
\hline Moderate-excellent & $42(89)$ \\
\hline \multicolumn{2}{|c|}{ Type of Primary Care Provider } \\
\hline Group & $28(60)$ \\
\hline Solo & $8(17)$ \\
\hline Walk-in & $3(6)$ \\
\hline Other & $2(4)$ \\
\hline \multicolumn{2}{|c|}{ Primary Care Network (PCN) } \\
\hline Yes & $12(26)$ \\
\hline No & $3(6)$ \\
\hline Unsure & $26(55)$ \\
\hline
\end{tabular}

\section{Results}

Overall, 1,685 individuals were approached by university students for this study of which 61 reported having asthma and completed the survey (Table 1). Of these, 47 lived in Alberta the majority being young adults in the 18 to 39 -year-old category (62\%), followed by $30 \%$ age 40 to $49,2 \%$ age 66 to 79 , and $6 \% 80$ and older. Most individuals were non- cigarette smokers

\begin{tabular}{|l|r|}
\hline Variable & $\mathrm{n}(\%)$ \\
\hline $\begin{array}{l}\text { NSAID-associated respiratory } \\
\text { disease }\end{array}$ & $3(6)$ \\
\hline $\begin{array}{l}\text { NICU Stay (neonatal intensive } \\
\text { care unit) }\end{array}$ & $5(11)$ \\
\hline Chronic Rhinosinusitis & $6(13)$ \\
\hline Chronic Urticarial & $2(4)$ \\
\hline Nasal Polyps (NP) & $2(4)$ \\
\hline $\begin{array}{l}\text { Acid Reflux: Gastro-Esophageal } \\
\text { Reflux Disease (GERD) }\end{array}$ & $6(13)$ \\
\hline Smoking (current) & $3(6)$ \\
\hline Smoking (past) & $11(23)$ \\
\hline Marijuana (current) & $4(9)$ \\
\hline Marijuana (past) & $7(15)$ \\
\hline $\begin{array}{l}\text { Immunotherapy/Allergy Shots } \\
\text { for Asthma Control (past) }\end{array}$ & $4(9)$ \\
\hline Puffer (current) & $1(2)$ \\
\hline $\begin{array}{l}\text { Inhaled Puffer Medication } \\
\text { (past) }\end{array}$ & 0 \\
\hline $\begin{array}{l}\text { †Pills for Asthma Control } \\
\text { (currently) }\end{array}$ & $42(85)$ \\
\hline $\begin{array}{l}\text { TPills for Asthma Control } \\
\text { (past) }\end{array}$ & $14(30)$ \\
\hline Biologics (currently) & $21(45)$ \\
\hline Biologics (past) & 0 \\
\hline Associated bronchiectasis & 0 \\
\hline $\begin{array}{l}\text { §Bronchopulmonary dys- } \\
\text { plasia (BPD) }\end{array}$ & \\
\hline Live on reserve & \\
\hline
\end{tabular}

tPills including singulair, prednisone, theophylline, antihistamines $\S B P D$ due to being born prematurely 
(77\% having never smoked), with $6 \%$ currently smoking. Some individuals were currently smoking marijuana $(4,9 \%)$, while $7(15 \%)$ had in the past

Pediatric history revealed that $11 \%$ of the population reported having a NICU stay. There was 1 subject with Bronchopulmonary Dysplasia (BPD) due to being born prematurely, and 3 (6\%) with Nonsteroidal Anti-Inflammatory drug (NSAID) associated respiratory disease.

The majority of individuals reported having a PCP; $87 \%(n=41)$. However, when asked if they see their PCP regularly (at least twice a year), that number was lower; $64 \%(n=30)$, with $n=10$ not visiting their PCP at least twice a year. Of those 10 individuals who do not see their PCP at least twice a year, 4 of them had 'uncontrolled asthma' as reported by either the ACT or the ACQ-5. Regarding type of PCP, $n=28$ were group practice, $n=8$ were solo practice, and $\mathrm{n}=3$ were walk-in clinics.

According to the ACQ-5, a score indicating "uncontrolled asthma" was reported for 32\% $(n=15)$ individuals. The ACT reported "uncontrolled asthma" in $36 \%(n=17)$ individuals. "Uncontrolled asthma" was reported in the ACT but not in the ACQ-5 6 times, while "uncontrolled asthma" was reported in the ACQ-5 but not in the ACT a total of 4 times. Asthma was uncontrolled in $45 \%(n=21)$ of subjects as quantified by either the ACQ-5 or ACT (Figure 1). Mean lifetime ED visits related to asthma were 4.5 , with a maximum of 50 for one individual. Mean lifetime hospitalizations related to asthma were 1.5 , while ICU stays were 0.25 Pulmonary physiology (i.e. spirometry / PFT) that proved asthma in the past 12 months was seen in $36 \%(n=17)$ of the patients. Only $55 \%(n=26)$ of individuals had ever had an airway challenge (i.e. methacholine, exercise challenge). Considering both physiologic testing and airway challenge, 70\% $(n=33)$ of the total self-reported asthma cases were confirmed by physiology and/or airway challenge. Subsequently, $30 \%(n=14)$ of the total self-reported

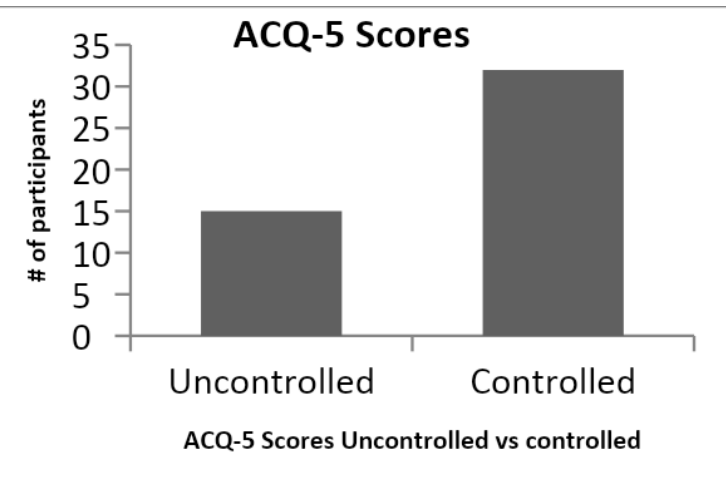

Figure 1 a: Asthma control measured through the ACQ-5.

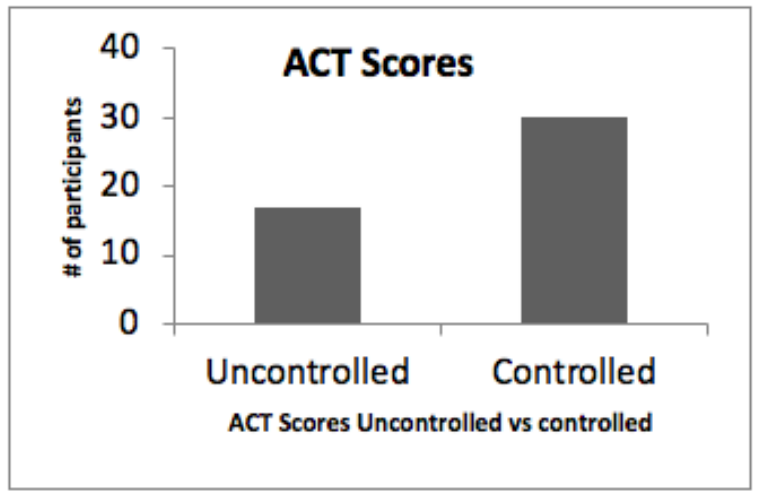

Figure $1 \mathrm{~b}$ : Asthma control measured through the ACT.

asthma cases did not have any physiologic testing or airway challenge to prove diagnosis.

Asthma quality of life measured through the mini-AQLQ showed $11 \% \quad(n=5)$ of individuals with poor-moderate QoL, with the majority having moderate-excellent QoL (Figure 2). Information was also collected on medication usage and co-morbidities. Systemic steroids were used by $28 \%$ of the population. $13 \%$ reported having chronic rhinosinusitis, and Gastro-Esophageal Reflux Disease (GERD). Chronic urticaria and nasal polyps were both uncommon (4\%).

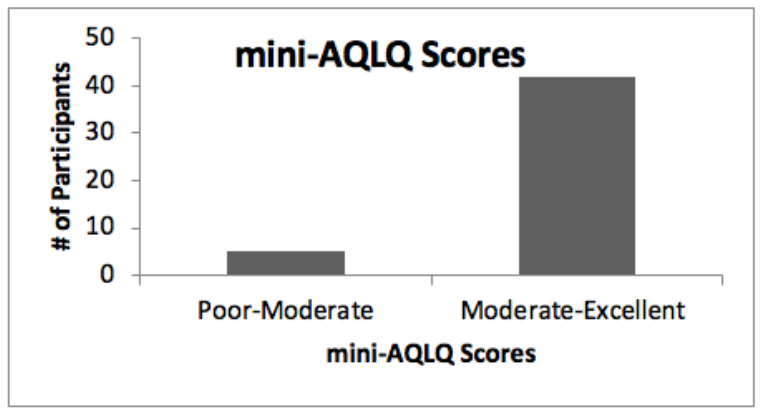

Figure 2: mini-AQLQ Scores 


\section{Discussion}

Despite there being numerous clinical guidelines, asthma control in Canada remains sub-optimal (FitzGerald, Boulet, Mclvor, Zimmerman, and Chapman, 2006). Our survey revealed that of those who had an asthma diagnosis ( $n=47), 45 \%$ $(n=21)$ did not have properly controlled asthma, as measured by either the ACQ-5 or ACT (Table 1 , Figure 1-2). At least $20 \%$ of those with asthma reported not visiting their PCP at least twice a year. Health care utilization is among the highest for chronic disease patients, where there is preventable overuse of emergency and other services. Though ED visits are declining in Alberta, asthma remains a major health and financial burden (Rosychuk, Youngson, and Rowe, 2015; Hollander, Kadlec, Hamdi, and Tessaro, 2009).

A 2009 study published in Healthcare Quarterly evaluated the Canadian health care system and noted that when "higher-care-needs patients" were linked to a PCP, overall healthcare costs declined, most notably in decreased hospital services (Hollander et al., 2009). The study determined that connection to a PCP was the strongest predictor of health care costs, even more significant than other variables (though this study did not specifically evaluate asthma).

Our findings regarding emergency and hospitalizations in Alberta raise some important questions regarding how asthma is managed in this population. Furthermore, a study determined that high continuity of care (thereby implying a strong patient-PCP relationship) reduced emergency visits by $60 \%-70 \%$, while reducing hospitalizations by about $25 \%$ (Cree, Bell, Johnson, and Carriere, 2006). The present study findings seem to confirm the $2015 \mathrm{ClHI}$ study, which found that $40 \%$ of Albertans do not have access to a strong $\mathrm{COC}$ model of primary care. Our study considered subjects' knowledge of primary care networks. Knowledge of PCNs was low, with over $50 \%$ of individuals not knowing whether their PCP was linked to one. Our study also revealed that of those with uncontrolled asthma, $62 \%(n=13)$ were unaware of their PCP being linked with a PCN or Local Health Integrated Network (LHIN). In a chronic disease such as asthma, PCNs can provide educational programs where action plans and management techniques are learned as preventative methods to achieve optimal asthma management. However, PCNs vary and not all have the same chronic disease focus. Respiratory education for patients with a chronic disease such as asthma is essential, and PCPs should provide proper and holistic education to their asthma patients.

Another Alberta study found that there were gaps in asthma education, written action plans as well as spirometry testing, where only $2 \%$ of asthma patients received an asthma action plan from their physician (Tsuyuki, Sin, Sharpe, Cowie, Nilsson, Paul Man, and Alberta Strategy to Help Manage Asthma [ASTHMA] Investigators, 2005). The little knowledge of PCNs in the current study raises concerns, given the nature of asthma being primarily self-managed. It is part of the PCP's responsibility to provide, through interdisciplinary programming an asthma education that incorporates a written asthma action plan. This programming is linked with a strong $\mathrm{COC}$ model where the patient has a strong and trusting relationship with their PCP. Our study also noted that of those with uncontrolled asthma as scored on the ACQ-5 or ACT, $67 \%(n=14)$, were in the 18-39-year-old age category, which may suggest a need for more education for this age group.

In Alberta, individuals do not need to enroll to see a family physician (Menec et al., 2006), which may often result in them seeing multiple physicians or walk-in clinics. The findings in this study show that most participants who did have a PCP, used traditional family practice clinic - group practice $(28,60 \%)$, with less using solo and walk in clinics (8, $17 \% ; 3,6 \%$ respectively). Of those with uncontrolled asthma in either the ACQ-5 or ACT, $52 \%(n=11)$ 
had a group PCP, while $33 \%(n=7)$ had either solo, walk-in or 'other' marked as their PCP. This raises concern for a potential group of asthma patients not receiving optimal continuity of care. In the context of relational continuity of care, ongoing and consistent use of a single PCP is especially important in chronic disease management (Menec et al, 2006). This aspect of continuity of care is essential for the patient to grow in their trust of PCPs and knowledge of which can greatly impact their asthma management and control.

A limitation in the present findings is the lack of rural Alberta data collection. These results may represent only part of the picture of asthma management in Alberta, as there may be many individuals living in remote locations where access to PCPs is sparse. Distance plays an important factor in regular access to PCPs, and certain areas of Alberta can also be associated with different levels of outdoor air pollution (Villeneuve, Chen, Rowe, and Coates, 2007). It is also clear that some First Nations communities in Alberta are in remote locations only accessible by flying in. Further collection of data is needed in rural areas, as well as reserves and far North communities. We did not capture gender or sex, as we did not think for this project on primary care it would be relevant. Another noteworthy limitation we must mention is that we did not capture psychiatric comorbidities including anxiety and depression, which are recognized as common comorbidities in asthma. This may also impact asthma control. It should also be mentioned that due to the requirement of internet connection for the administration of the web-based survey, many locations were excluded from potential recruitment.

While the sample size for this study is lower than anticipated, it is evident that asthma is still not optimally controlled, and a considerable percentage of subjects were unsure about PCNs while utilizing ED and hospital services. This study raises questions about continuity of care and the utilization of primary care for adults who have asthma. The importance of primary care is critical in managing patients with asthma and must be emphasized, particularly since it is a chronic disease (Chung, Johnson, and Summers, 2018). Further work should include comprehensive data on primary care by health jurisdiction within Canada. This is particularly relevant in Canada given its single payer system (funded through taxpayer dollars).

\section{Acknowledgments}

The authors would like to thank Ms. Iris de Guzman for her administrative support, the following students who spent a few weeks recruiting subjects as they learned about research (Hailey Hitchings, Lloyd Visser and Shaina Corrick). We would also like to thank Dr. Maeve Smith MD, FRCPC, (Respirology) and Dr. Raj Padwal, MD, FRCPC (General Internist) for their study design input, as well as Dr. Carolyn Ross, BScN, RN, PhD (Faculty of Nursing) and Mrs. Jillian Peters, BScN, RN (Faculty of Nursing) for their help throughout this project.

\section{Declaration of Interests}

The authors have no conflict of interests to note. 


\section{References}

Bateman, E. D., Hurd, S. S., Barnes, P. J., Bousquet, J., Drazen, J. M., FitzGerald, M., ... \& Pizzichini, E. (2008). Global strategy for asthma management and prevention: GINA executive summary. European Respiratory Journal, 31(1), 143-78.

Canadian Institute for Health Information. Continuity of Care With Family Medicine Physicians: Why It Matters. Ottawa, ON: CIHI; 2015. Available from: https://secure.cihi.ca/free_products/UPC_ ReportFINAL_EN.pdf

Christakis, D. A., Mell, L., Koepsell, T. D., Zimmerman, F. J., \& Connell, F. A. (2001). Association of lower continuity of care with greater risk of emergency department use and hospitalization in children. Pediatrics, 107(3), 524-529.

Chung, L. P., Johnson, P., \& Summers, Q. (2018). Models of care for severe asthma: the role of primary care. Medical Journal of Australia, 209(S2), S34-S40.

Cree, M., Bell, N. R., Johnson, D., \& Carriere, K. C. (2006). Increased continuity of care associated with decreased hospital care and emergency department visits for patients with asthma. Disease Management, 9(1), 63-71.

FitzGerald, J. M., Boulet, L. P., Mclvor, R. A., Zimmerman, S., \& Chapman, K. R. (2006). Asthma control in Canada remains suboptimal: the Reality of Asthma Control (TRAC) study. Canadian Respiratory Journal, 13(5), 253-259.

Goeman, D., Jenkins, C., Crane, M., Paul, E., \& Douglass, J. (2013). Educational intervention for older people with asthma: a randomised controlled trial. Patient Education and Counseling, 93(3), 586595.

Haggerty, J. L., Reid, R. J., Freeman, G. K., Starfield, B. H., Adair, C. E., \& McKendry, R. (2003). Continuity of care: a multidisciplinary review. BMJ, 327(7425), 1219-1221.

Hollander, M. J., \& Kadlec, H. (2015). Financial implications of the continuity of primary care. The Permanente Journal, 19(1), 4.

Hollander, M. J., Kadlec, H., Hamdi, R., \& Tessaro, A. (2009). Increasing value for money in the Canadian healthcare system: new findings on the contribution of primary care services. Healthcare Quarterly (Toronto, Ont.), 12(4), 32-44.

Jia, C. E., Zhang, H. P., Lv, Y., Liang, R., Jiang, Y. Q., Powell, H., ... \& Wang, G. (2013). The Asthma Control Test and Asthma Control Questionnaire for assessing asthma control: systematic review and meta-analysis. Journal of Allergy and Clinical Immunology, 131(3), 695-703.

Juniper, E. F., O' byrne, P. M., Guyatt, G. H., Ferrie, P. J., \& King, D. R. (1999). Development and validation of a questionnaire to measure asthma control. European Respiratory Journal, 14(4), 902-907.

Juniper, E. F., Svensson, K., Mörk, A. C., \& Ståhl, E. (2005). Measurement properties and interpretation of three shortened versions of the asthma control questionnaire. Respiratory Medicine, 99(5), 553558.

Kaplan, A. (2008). Systems for the management of respiratory disease in primary care-an international series: Canada. Primary Care Respiratory Journal, 17(2), 73.

Lougheed, M. D., Lemière, C., Dell, S. D., Ducharme, F. M., FitzGerald, J. M., Leigh, R., Licskai, C., Rowe, B.H., Bowie, D., Becker, A., \& Boulet, L. P. (2010). Consensus Summary for children six years of age and over, and adults. Canadian Respiratory Journal, 17(1), 15-24.

Love, M. M., Mainous, A. G. I., Talbert, J. C., \& Hager, G. L. (2000). Continuity of care and the physicianpatient relationship. Journal of Family Practice, 49(11), 998-998.

Menec, V. H., Sirski, M., Attawar, D., \& Katz, A. (2006). Does continuity of care with a family physician 
reduce hospitalizations among older adults? Journal of Health Services Research \& Policy, 11(4), 196-201.

Nathan, R. A., Sorkness, C. A., Kosinski, M., Schatz, M., Li, J. T., Marcus, P., .. \& Pendergraft, T. B. (2004). Development of the asthma control test: a survey for assessing asthma control. Journal of Allergy and Clinical Immunology, 113(1), 59-65.

Reddel, H. K., Taylor, D. R., Bateman, E. D., Boulet, L. P., Boushey, H. A., Busse, W. W., ... \& de Jongste, J. C. (2009). An official American Thoracic Society/European Respiratory Society statement: asthma control and exacerbations: standardizing endpoints for clinical asthma trials and clinical practice. American Journal of Respiratory and Critical Care Medicine, 180(1), 59-99.

Ridd, M., Shaw, A., Lewis, G., \& Salisbury, C. (2009). The patient-doctor relationship: a synthesis of the qualitative literature on patients' perspectives. British Journal of General Practice, 59(561), e116-e133.

Rosychuk, R. J., Youngson, E., \& Rowe, B. H. (2015). Presentations to Alberta emergency departments for asthma: a time series analysis. Academic Emergency Medicine, 22(8), 942-949.

Schatz, M., Sorkness, C. A., Li, J. T., Marcus, P., Murray, J. J., Nathan, R. A., ... \& Jhingran, P. (2006). Asthma Control Test: reliability, validity, and responsiveness in patients not previously followed by asthma specialists. Journal of Allergy and Clinical Immunology, 117(3), 549-556.

Statistics Canada. Population, urban and rural, by province and territory (Alberta) [Internet]. 2011 [cited 17 Oct 2019]. Available from: https://www.statcan.gc.ca/tables-tableaux/sum-som/l01/cst01/ demo62j-eng.htm

Tsuyuki, R. T., Sin, D. D., Sharpe, H. M., Cowie, R. L., Nilsson, C., Paul Man, S. F., \& Alberta Strategy to Help Manage Asthma (ASTHMA) Investigators. (2005). Management of asthma among communitybased primary care physicians. Journal of Asthma, 42(3), 163-167.

Villeneuve, P. J., Chen, L., Rowe, B. H., \& Coates, F. (2007). Outdoor air pollution and emergency department visits for asthma among children and adults: a case-crossover study in northern Alberta, Canada. Environmental Health, 6(1), 40. 